\title{
A renormalized expression for the turbulent energy dissipation rate
}

\author{
C. Johnston and W. D. McComb* \\ Department of Physics and Astronomy, University of Edinburgh, Edinburgh, EH9 3JZ
}

(October 27, 2018)

\begin{abstract}
Conditional elimination of degrees of freedom is shown to lead to an exact expression for the rate of turbulent energy dissipation in terms of a renormalized viscosity and a correction. The correction is neglected on the basis of a previous hypothesis [1] that there is a range of parameters for which a quasi-stochastic estimate is a good approximation to the exact conditional average. This hypothesis was tested by a perturbative calculation to second order in the local Reynolds number, and the Kolmogorov prefactor (taken as a measure of the renormalized dissipation rate) was found to reach a fixed point which was insensitive to initial values of the kinematic viscosity and to values of the spatial rescaling factor $h$ in the range $0.4 \leq h \leq 0.8$.

47.27.Ak, 47.27.Eq, 47.27.Gs, 05.20.-y
\end{abstract}

Typeset using REVTEX

*email: W.D.McComb@ed.ac.uk 
In the numerical simulation of fluid turbulence, as in other areas of computational physics, there is a practical requirement to reduce the number of degrees of freedom explicitly simulated. However, turbulence has the noteworthy requirement that any such reduction must maintain the rate at which energy is dissipated. In this letter we put forward a method of renormalizing the energy dissipation rate.

It seems to be widely understood that any attempt to reduce the number of degrees of freedom in the theoretical description of fluid turbulence requires some form of conditional average, in which the retained modes are kept unaveraged [2]. Yet this requirement has not been recognised in most attempts to apply the dynamical Renormalization Group (RG) algorithm to turbulence. Normally such methods rely instead on a band-filtered unconditional average. A critical appraisal of some of the leading approaches in this area will be found in the paper by Eyink [3].

Originally, our own work, although introducing some features of the conditional average, also relied on the use of the band-filtered unconditional average [圆]. Later it was recognized that a conditional average in turbulence can only be carried out as an approximation, and the two-field decomposition was introduced to separate out random and deterministic effects [5.6]. Recently, we have redefined the conditional average in the form of a limit, eliminating the need to separate into two fields [1].

The development reported here is that one of the corrections (to mode elimination) in the momentum equation, vanishes identically in the energy equation (and hence does not contribute to energy transfer), while a second correction contributes to the energy spectrum but vanishes identically in the equation for the dissipation rate.

We consider homogeneous, isotropic, incompressible, stationary turbulence, with dissipation rate $\varepsilon$ given by

$$
\varepsilon=\int_{0}^{\infty} \mathrm{d} k 2 \nu_{0} k^{2} E(k) \simeq \int_{0}^{k_{0}} \mathrm{~d} k 2 \nu_{0} k^{2} E(k),
$$

where the approximate equality defines the maximum wavenumber $k_{0}$. The value of $k_{0}$ is of the same order as the Kolmogorov dissipation wavenumber $k_{d}^{(0)}=\left(\varepsilon / \nu_{0}^{3}\right)^{1 / 4}$, where $\nu_{0}$ is the 
kinematic viscosity.

In such turbulence the pair-correlation takes the form $\left\langle u_{\alpha}(\boldsymbol{k}, t) u_{\beta}\left(\boldsymbol{k}^{\prime}, s\right)\right\rangle=$ $D_{\alpha \beta}(\boldsymbol{k}) Q(\boldsymbol{k} ; t, s) \delta\left(\boldsymbol{k}+\boldsymbol{k}^{\prime}\right)$, where $D_{\alpha \beta}(\boldsymbol{k})=\delta_{\alpha \beta}-k_{\alpha} k_{\beta} / k^{2}$, and the energy spectrum is related to the spectral density by $E(k, t)=4 \pi k^{2} Q(k ; t, t)$. To complete the specification of our problem, we assume that energy is being injected into some low range of wavenumbers by a source term $W(k)$, which satisfies

$$
\int_{0}^{\kappa} \mathrm{d} k W(k)=\varepsilon
$$

for some $\kappa \ll k_{d}^{(0)}$. This ensures stationarity.

Next we introduce a version of the $R G$ which leads to a renormalized dissipation rate equation. The Navier-Stokes equation (NSE) may be written in dimensionless form as:

$$
\begin{aligned}
& \left\{\partial_{\hat{t}}+\hat{\nu}_{0}(\hat{k}) \hat{k}^{2}\right\} \hat{u}_{\alpha}(\hat{\boldsymbol{k}}, \hat{t})= \\
& \quad=R_{0}\left(k_{0}\right) M_{\alpha \beta \gamma}(\hat{\boldsymbol{k}}) \int \mathrm{d}^{3} \hat{j} \hat{u}_{\beta}(\hat{\boldsymbol{j}}, \hat{t}) \hat{u}_{\gamma}(\hat{\boldsymbol{k}}-\hat{\boldsymbol{j}}, \hat{t}),
\end{aligned}
$$

on $0<\hat{k}<\hat{k}_{0}=1$, where $\hat{k}=k / k_{0}, \hat{t}=t / \tau\left(k_{0}\right), \hat{u}_{\alpha}(\hat{\boldsymbol{k}}, t)=u_{\alpha}(\boldsymbol{k}, t) / V\left(k_{0}\right), \tau\left(k_{0}\right)$ is an, as yet, undefined timescale, $V\left(k_{0}\right)$ is the r.m.s. value of a velocity mode with $|\boldsymbol{k}|=k_{0}$, defined for any $k$ by

$$
V^{2}(k)=\frac{1}{k^{3}}\left\langle u_{\alpha}(\boldsymbol{k}, t) u_{\alpha}(-\boldsymbol{k}, t)\right\rangle,
$$

$R_{0}\left(k_{0}\right)=\tau\left(k_{0}\right) V\left(k_{0}\right) k_{0}^{4}$ is the local Reynolds number (see Batchelor [7], p107) and $M_{\alpha \beta \gamma}(\boldsymbol{k})=$ $(2 i)^{-1}\left[k_{\beta} D_{\alpha \gamma}(\boldsymbol{k})+k_{\gamma} D_{\alpha \beta}(\boldsymbol{k})\right]$. It should also be noted that the local Reynolds number is indeed non-dimensional, since $u_{\alpha}(\mathbf{k}, \mathbf{t})$ has dimensions $L^{4} T^{-1}$. The dynamical RG algorithm can now be stated as follows:

1. Rescale all wavevectors on $\hat{k}_{1}\left(=h \hat{k}_{0}\right)$, where $0<h<1$, for example $k^{\prime}=\hat{k} / \hat{k}_{1}$, such that $0<k^{\prime}<h^{-1}$, and then average out the effects of the high wavenumber modes to obtain a dynamical equation for the modes on the interval $0<k^{\prime}<k_{1}^{\prime}(\equiv 1)$.

2. Use this low-wavenumber NSE to obtain the low-wavenumber energy balance equation. 
3. Integrate the energy balance equation with respect to $k^{\prime}$ up to the value $k^{\prime}=k_{1}^{\prime}(\equiv 1)$ to derive an equation for the dissipation rate.

4. Repeat these steps until the dissipation rate reaches a fixed point, at a new maximum wavenumber $k_{N}^{\prime}=h^{N} k_{0}^{\prime}$.

The first step is to write the velocity fields as

$$
\hat{u}_{\alpha}^{ \pm}(\hat{\boldsymbol{k}}, \hat{t})=V\left(k_{1}\right) \psi_{\alpha}^{ \pm}\left(\boldsymbol{k}^{\prime}, t^{\prime}\right)
$$

where $\psi_{\alpha}^{-}\left(\boldsymbol{k}^{\prime}, t^{\prime}\right)$ is defined on $0<k^{\prime}<1$ and $\psi_{\alpha}^{+}\left(\boldsymbol{k}^{\prime}, t^{\prime}\right)$ is defined on $1<k^{\prime}<h^{-1}$. The combined (low- $k$ and high- $k$ ) equation of motion then takes the form:

$$
\begin{aligned}
& \left\{\partial_{t^{\prime}}+\nu_{0}^{\prime}\left(k^{\prime}\right) k^{\prime 2}\right\} \psi_{\alpha}^{ \pm}\left(\boldsymbol{k}^{\prime}, t^{\prime}\right)= \\
& =R_{1}\left(k_{1}\right) M_{\alpha \beta \gamma}^{ \pm}\left(\mathbf{k}^{\prime}\right) \int \mathrm{d}^{3} \mathbf{j}^{\prime} \psi_{\beta}\left(\boldsymbol{j}^{\prime}, \mathbf{t}^{\prime}\right) \psi_{\gamma}\left(\boldsymbol{k}^{\prime}-\boldsymbol{j}^{\prime}, \mathbf{t}^{\prime}\right),
\end{aligned}
$$

where $R_{1}\left(k_{1}\right)=\tau\left(k_{1}\right) V\left(k_{1}\right) k_{1}^{4}$. Next we average out the effect of the high- $k$ modes, while leaving the low- $k$ modes unaffected. In general this will require the conditional projection of some functional $\mathcal{F}\left[\psi_{\alpha}\right]$ on the $\psi_{\alpha}^{-}$, which we denote by a subscript ' $c$ ', viz. $\langle\cdot\rangle_{c}$. This should not be confused with the usual ensemble average, as denoted by $\langle\cdot\rangle$. An important property of the conditional average is that the constraint is lifted by a further unconditional average [8].

Taking the low- $k$ equation, as given by (6), we conditionally average both sides, and decompose the right-hand side according to (5), to obtain:

$$
\begin{aligned}
& \left\{\partial_{t^{\prime}}+\nu_{0}^{\prime}\left(k^{\prime}\right) k^{\prime 2}\right\} \psi_{\alpha}^{-}\left(\boldsymbol{k}^{\prime}\right)= \\
& =R_{1}\left(k_{1}\right) M_{\alpha \beta \gamma}^{-}\left(\boldsymbol{k}^{\prime}\right) \int \mathrm{d}^{3} j^{\prime}\left\{\left\langle\psi_{\beta}^{-}\left(\boldsymbol{j}^{\prime}\right) \psi_{\gamma}^{-}\left(\boldsymbol{k}^{\prime}-\boldsymbol{j}^{\prime}\right)\right\rangle_{c}+\right. \\
& \left.+2\left\langle\psi_{\beta}^{-}\left(\boldsymbol{j}^{\prime}\right) \psi_{\gamma}^{+}\left(\boldsymbol{k}^{\prime}-\boldsymbol{j}^{\prime}\right)\right\rangle_{c}+\left\langle\psi_{\beta}^{+}\left(\boldsymbol{j}^{\prime}\right) \psi_{\gamma}^{+}\left(\boldsymbol{k}^{\prime}-\boldsymbol{j}^{\prime}\right)\right\rangle_{c}\right\} .
\end{aligned}
$$

We shall see presently that only the last term on the RHS of (7) contributes to the energy dissipation rate. In [1] we gave a method for the approximate calculation of this conditional average. We shall refer to this approximation as the quasi-stochastic estimate (QSE) of 
the conditional average and denote it by $\langle\cdot\rangle_{Q S E}$. Then, rearranging $(7)$ and adding and subtracting quantities to leave it unaffected, we may write the low-wavenumber equation as:

$$
\begin{aligned}
& \left(\partial_{t^{\prime}}+\nu_{0}^{\prime}\left(k^{\prime}\right) k^{\prime 2}\right) \psi_{\alpha}^{-}\left(\boldsymbol{k}^{\prime}\right)- \\
& -R_{1}\left(k_{1}\right) M_{\alpha \beta \gamma}^{-}\left(\boldsymbol{k}^{\prime}\right) \int \mathrm{d}^{3} j^{\prime}\left\langle\psi_{\beta}^{+}\left(\boldsymbol{j}^{\prime}\right) \psi_{\gamma}^{+}\left(\boldsymbol{k}^{\prime}-\boldsymbol{j}^{\prime}\right)\right\rangle_{Q S E} \\
& =R_{1}\left(k_{1}\right) M_{\alpha \beta \gamma}^{-}\left(\boldsymbol{k}^{\prime}\right) \int \mathrm{d}^{3} j^{\prime} \psi_{\beta}^{-}\left(\boldsymbol{j}^{\prime}\right) \psi_{\gamma}^{-}\left(\boldsymbol{k}^{\prime}-\boldsymbol{j}^{\prime}\right)+ \\
& \quad+S_{\alpha}^{-}\left(\boldsymbol{k}^{\prime} \mid k_{1}^{\prime}\right)
\end{aligned}
$$

where

$$
\begin{aligned}
& S_{\alpha}^{-}\left(\boldsymbol{k}^{\prime} \mid k_{1}^{\prime}\right)=R_{1}\left(k_{1}\right) M_{\alpha \beta \gamma}^{-}\left(\boldsymbol{k}^{\prime}\right) \times \\
& \times \int \mathrm{d}^{3} j^{\prime}\{\underbrace{\left\langle\psi_{\beta}^{-}\left(\boldsymbol{j}^{\prime}\right) \psi_{\gamma}^{-}\left(\boldsymbol{k}^{\prime}-\boldsymbol{j}^{\prime}\right)\right\rangle_{c}-\psi_{\beta}^{-}\left(\boldsymbol{j}^{\prime}\right) \psi_{\gamma}^{-}\left(\boldsymbol{k}^{\prime}-\boldsymbol{j}^{\prime}\right)}_{S_{1}}+ \\
& +2 \underbrace{\left\langle\psi_{\beta}^{-}\left(\boldsymbol{j}^{\prime}\right) \psi_{\gamma}^{+}\left(\boldsymbol{k}^{\prime}-\boldsymbol{j}^{\prime}\right)\right\rangle_{c}}_{S_{2}}+ \\
& +\underbrace{\left\langle\psi_{\beta}^{+}\left(\boldsymbol{j}^{\prime}\right) \psi_{\gamma}^{+}\left(\boldsymbol{k}^{\prime}-\boldsymbol{j}^{\prime}\right)\right\rangle_{c}-\left\langle\psi_{\beta}^{+}\left(\boldsymbol{j}^{\prime}\right) \psi_{\gamma}^{+}\left(\boldsymbol{k}^{\prime}-\boldsymbol{j}^{\prime}\right)\right\rangle_{Q S E}}_{S_{3}}\} .
\end{aligned}
$$

We now wish to obtain the QSE, $\left\langle\psi_{\beta}^{+}\left(\boldsymbol{j}^{\prime}, t^{\prime}\right) \psi_{\gamma}^{+}\left(\boldsymbol{k}^{\prime}-\boldsymbol{j}^{\prime}, t^{\prime}\right)\right\rangle_{Q S E}$, which appears on the LHS of (8). Following the procedure in [1], we form an equation of motion for this quantity from (6). This is solved perturbatively as a power series in $R\left(k_{1}\right)$ and the band-filtered moments of the $\psi^{+}$. As a result, the low- $k$ equation, after eliminating the first band of modes, takes the form

$$
\begin{aligned}
& \left\{\partial_{t^{\prime}}+\nu_{0}^{\prime}\left(k^{\prime}\right) k^{\prime 2}\right\} \psi_{\alpha}^{-}\left(\boldsymbol{k}^{\prime}\right)-\int \mathrm{d} s^{\prime} A_{\alpha \beta}\left(\boldsymbol{k}^{\prime}, t^{\prime}-s^{\prime}\right) \psi_{\beta}^{-}\left(\boldsymbol{k}^{\prime}, s^{\prime}\right) \\
& =R_{1}\left(k_{1}\right) M_{\alpha \beta \gamma}^{-}\left(\boldsymbol{k}^{\prime}\right) \int \mathrm{d}^{3} j^{\prime}\left\langle\psi_{\beta}^{-}\left(\boldsymbol{j}^{\prime}\right) \psi_{\gamma}^{-}\left(\boldsymbol{k}^{\prime}-\boldsymbol{j}^{\prime}\right)\right\rangle_{c} \\
& \quad+S_{\alpha}^{-}\left(\boldsymbol{k}^{\prime} \mid k_{1}^{\prime}\right),
\end{aligned}
$$

where

$$
\begin{aligned}
& A_{\alpha \beta}\left(\boldsymbol{k}^{\prime}, t^{\prime}-s^{\prime}\right)=D_{\alpha \beta}\left(\boldsymbol{k}^{\prime}\right) R_{1}^{2}\left(k_{1}\right) \times \\
& \times\left[A^{(0)}\left(k^{\prime}, t^{\prime}-s^{\prime}\right)+A^{(1)}\left(k^{\prime}, t^{\prime}-s^{\prime}\right) R_{1}\left(k_{1}\right)+\right. \\
& \left.\quad+A^{(2)}\left(k^{\prime}, t^{\prime}-s^{\prime}\right) R_{1}^{2}\left(k_{1}\right)+\ldots\right] .
\end{aligned}
$$


The coefficients $A^{(0)}, A^{(1)}, A^{(2)}, \ldots$ depend on the moments of $\psi^{+}$of order $2,3,4, \ldots$ respectively. It should also be noted that the even-order coefficients are real and the odd-order are imaginary, since the expansion is effectively in powers of $M_{\alpha \beta \gamma}(\boldsymbol{k})$, which is imaginary.

We now form the energy balance equation for the explicit scales $k \leq k_{1}$, by multiplying each side of (10) through by $\psi_{\alpha}^{-}\left(-\boldsymbol{k}^{\prime}, t^{\prime}\right)$ and averaging unconditionally. We then multiply through by appropriate factors to restore the original unscaled variables, in the process introducing the energy spectrum $E(k)$. We also add $W(k)$, as specified in $(2)$, with the result:

$$
\begin{aligned}
& \left(\partial_{t}+2 \nu_{0}(k) k^{2}\right) E(k)+2 \int \mathrm{d} s A(k, t-s) E(k, s)= \\
& =W(k)+T(k)+8 \pi k^{2} V^{2}\left(k_{1}\right)\left\langle S_{\alpha}^{-}\left(\boldsymbol{k} \mid k_{1}\right) \psi_{\alpha}^{-}(-\boldsymbol{k})\right\rangle,
\end{aligned}
$$

where $A(k)=\operatorname{tr} A_{\alpha \beta}(\boldsymbol{k})$ and $T(k, t)=\int \mathrm{d} j \tilde{T}(k, j,|\boldsymbol{k}-\boldsymbol{j}| ; t)$ is the usual transfer spectrum, with wavenumbers in the interval $0 \leq k, j,|\boldsymbol{k}-\boldsymbol{j}| \leq k_{1}$.

Lastly, we may form an equation for the rate at which energy is transferred through the modes of the system. Integrating (12) with respect to $k$, we obtain

$$
\begin{aligned}
& 2 \int_{0}^{k_{1}} \mathrm{~d} k\left[\nu_{0} k^{2}+A(k)\right] E(k)=\varepsilon+ \\
& +8 \pi \int_{0}^{k_{1}} \mathrm{~d} k k^{2} V^{2}\left(k_{1}\right)\left\langle S_{\alpha}^{-}\left(\boldsymbol{k} \mid k_{1}\right) \psi_{\alpha}^{-}(-\boldsymbol{k})\right\rangle .
\end{aligned}
$$

Note that the integral over the transfer term vanishes identically due to the antisymmetry of $\tilde{T}(k, j,|\boldsymbol{k}-\boldsymbol{j}| ; t)$ under interchange of $\boldsymbol{k}$ and $\boldsymbol{j}$ (see [7], p85).

At this stage, all three renormalized conservation equations (for momentum, energy and dissipation rate) are exact. Now consider the effect of the term $S_{\alpha}^{-}$, divided into three parts, as shown in (9), and begin with the energy equation. The conditional average behaves as a stochastic variable under a further unconditional average [8]. Thus for the first term we have (schematically)

$$
\begin{aligned}
\left\langle S_{1} \psi_{\alpha}^{-}\right\rangle \sim \int & \mathrm{d}^{3} j^{\prime}\left\{\left\langle\left\langle\psi_{\beta}^{-}\left(\boldsymbol{j}^{\prime}\right) \psi_{\gamma}^{-}\left(\boldsymbol{k}^{\prime}-\boldsymbol{j}^{\prime}\right)\right\rangle_{c} \psi_{\alpha}^{-}\left(-\boldsymbol{k}^{\prime}\right)\right\rangle-\right. \\
- & \left.\left\langle\psi_{\beta}^{-}\left(\boldsymbol{j}^{\prime}\right) \psi_{\gamma}^{-}\left(\boldsymbol{k}^{\prime}-\boldsymbol{j}^{\prime}\right) \psi_{\alpha}^{-}\left(-\boldsymbol{k}^{\prime}\right)\right\rangle\right\}=0
\end{aligned}
$$


and so the contribution from $S_{1}$ vanishes identically in the energy equation.

Now we turn to the dissipation equation: evidently we need only consider $S_{2}$ and $S_{3}$. The first of these gives

$$
\begin{aligned}
& \int \mathrm{d}^{3} k^{\prime}\left\langle S_{2} \psi_{\alpha}^{-}\right\rangle \sim \\
& \sim \int \mathrm{d}^{3} k^{\prime} \int \mathrm{d}^{3} j^{\prime}\left\langle\psi_{\beta}^{-}\left(\boldsymbol{j}^{\prime}\right) \psi_{\gamma}^{+}\left(\boldsymbol{k}^{\prime}-\boldsymbol{j}^{\prime}\right) \psi_{\alpha}^{-}\left(-\boldsymbol{k}^{\prime}\right)\right\rangle=0,
\end{aligned}
$$

by antisymmetry under interchange of $\boldsymbol{k}^{\prime}$ and $\boldsymbol{j}^{\prime}$. It should be noted that this property holds only because both wavenumbers are on the same interval. This is not the case regarding the contribution from $S_{3}$, which is

$$
\begin{aligned}
& \int \mathrm{d}^{3} k^{\prime}\left\langle S_{3} \psi_{\alpha}^{-}\right\rangle \sim \\
& \sim \int \mathrm{d}^{3} k^{\prime} \int \mathrm{d}^{3} j^{\prime}\left\{\left\langle\psi_{\beta}^{+}\left(\boldsymbol{j}^{\prime}\right) \psi_{\gamma}^{+}\left(\boldsymbol{k}^{\prime}-\boldsymbol{j}^{\prime}\right) \psi_{\alpha}^{-}\left(-\boldsymbol{k}^{\prime}\right)\right\rangle-\right. \\
& \left.\quad-\left\langle\psi_{\beta}^{+}\left(\boldsymbol{j}^{\prime}\right) \psi_{\gamma}^{+}\left(\boldsymbol{k}^{\prime}-\boldsymbol{j}^{\prime}\right) \psi_{\alpha}^{-}\left(-\boldsymbol{k}^{\prime}\right)\right\rangle_{Q S E}\right\} .
\end{aligned}
$$

However, note that the two terms will cancel under any circumstances in which the QSE is a good model for the exact conditional average.

Now, in order to perform an RG-style iteration, we truncate the expansion for $A_{\alpha \beta}$, as given by (11), at lowest order and rename

$$
R_{1}^{2}\left(k_{1}\right) A^{(0)}\left(k^{\prime}, 0\right)=\delta \nu_{0}\left(k^{\prime}\right) k^{\prime 2} .
$$

Note that $A^{(1)}$ is imaginary and therefore cannot contribute to the dissipation rate. This means that we effectively neglect terms of order $R_{1}^{4}\left(k_{1}\right)$ and higher. To this level of approximation, (10) can be written as

$$
\begin{aligned}
& \left(\partial_{t^{\prime}}+\nu_{1}^{\prime}\left(k^{\prime}\right) k^{\prime 2}\right) \psi_{\alpha}^{-}\left(\boldsymbol{k}^{\prime}\right)=S_{\alpha}^{-}\left(\boldsymbol{k}^{\prime} \mid k_{1}^{\prime}\right)+ \\
& \quad+R_{1}\left(k_{1}\right) M_{\alpha \beta \gamma}^{-}\left(\boldsymbol{k}^{\prime}\right) \int \mathrm{d}^{3} j^{\prime} \psi_{\beta}^{-}\left(\boldsymbol{j}^{\prime}\right) \psi_{\gamma}^{-}\left(\boldsymbol{k}^{\prime}-\boldsymbol{j}^{\prime}\right),
\end{aligned}
$$

for $0<k^{\prime}<1$. The renormalized viscosity is given by

$$
\nu_{1}^{\prime}\left(k^{\prime}\right)=\nu_{0}^{\prime}\left(k^{\prime}\right)+\delta \nu_{0}^{\prime}\left(k^{\prime}\right)
$$


and the equation for the increment is

$$
\begin{aligned}
\delta \nu_{0}^{\prime}\left(k^{\prime}\right)= & R_{1}^{2}\left(k_{1}\right) \lim _{\ell^{\prime} \rightarrow h^{-1}} \Delta_{0}\left(k^{\prime}\right)\left[\frac{\left(k^{\prime 2} / 2\right)+j^{\prime 2}-k^{\prime} j^{\prime} \mu}{j^{\prime 2}-k^{\prime} j^{\prime} \mu}\right] \\
& +\mathcal{O}\left(R_{1}^{4}\left(k_{1}\right)\right)
\end{aligned}
$$

where $\mu$ is the cosine of the angle between $\boldsymbol{k}^{\prime}$ and $\boldsymbol{j}^{\prime}$ and $\Delta_{0}\left(k^{\prime}\right)$ is the two-field form of the increment [6], given by

$$
\Delta_{0}\left(k^{\prime}\right)=\frac{1}{k^{\prime 2}} \int \mathrm{d}^{3} j^{\prime} \frac{L\left(\boldsymbol{k}^{\prime}, \boldsymbol{j}^{\prime}\right) \hat{Q}^{+}\left(\ell^{\prime}\right)}{\nu_{0}^{\prime}\left(j^{\prime}\right) j^{\prime 2}+\nu_{0}^{\prime}\left(\ell^{\prime}\right) \ell^{\prime 2}} .
$$

Here $\ell^{\prime}=\left|\boldsymbol{k}^{\prime}-\boldsymbol{j}^{\prime}\right|, 1<k^{\prime}, \ell^{\prime}<h^{-1}$ and $L\left(\boldsymbol{k}^{\prime}, \boldsymbol{j}^{\prime}\right)=-2 M_{\delta \beta \gamma}^{-}\left(\boldsymbol{k}^{\prime}\right) M_{\beta \delta \epsilon}^{+}\left(\boldsymbol{j}^{\prime}\right) D_{\epsilon \gamma}^{+}\left(\boldsymbol{k}^{\prime}-\boldsymbol{j}^{\prime}\right)$. Equation (20) for the increment to the viscosity involves $\lim \left|\boldsymbol{k}^{\prime}-\boldsymbol{j}^{\prime}\right| \rightarrow h^{-1}$ (see [1]), and if this limit is evaluated by taking the $\hat{Q}^{+}\left(\left|\boldsymbol{k}^{\prime}-\boldsymbol{j}^{\prime}\right|\right)$ as an expansion in Taylor series about $k^{\prime}=1$, then we make contact with the two-field version of McComb and Watt [6], with spectral density given by

$$
\hat{Q}\left(l^{\prime}\right)=\frac{1}{k_{1} V^{2}\left(k_{1}\right)}\left\{h^{11 / 3}-\frac{11}{3} h^{14 / 3}\left(l^{\prime}-h^{-1}\right)\right\} .
$$

It should be noted however that the factor in square brackets in (20) is new. This arises because we were able to improve on the Markovian approximation used in the earlier calculations of McComb et al. [4].6]. Details of this analysis will be given in a subsequent paper. The equations for any iteration labelled $n$ can be found by induction, and numerical calculation shows that the renormalized viscosity approaches a fixed point for some $n=N$, where in practice $N=5$ or 6 , for most values of the spatial rescaling factor. At the fixed point, (13) may be rearranged to give

$$
\begin{aligned}
\varepsilon= & 2 \int_{0}^{k_{N}} \mathrm{~d} k \nu_{N}(k) k^{2} E(k)- \\
& -8 \pi \int_{k_{N-1}}^{k_{N}} \mathrm{~d} k k^{2} V^{2}\left(k_{N}\right)\left\langle S_{3} \psi_{\alpha}^{-}(-\boldsymbol{k})\right\rangle,
\end{aligned}
$$

where $\left\langle S_{3} \psi_{\alpha}^{-}(-\boldsymbol{k})\right\rangle$ is shown schematically in equation (16).

Let us now consider how to assess this work. We begin by noting that the renormalized 'viscous term' in (18) is not an observable, even though it may be calculated to any order 
using (11). This is because $S_{\alpha}^{-}$varies from realization to realization of the explicit scales. In contrast, the renormalized viscosity in (12) would be (if we took the step given in (17)) an observable, as all terms in the equation have been averaged. However, the contribution from $S_{2}$ is likely to prove important for energy transfer and, as this has been omitted from our calculation, we shall leave discussion of this to a fuller account, and concentrate on (23) for the dissipation rate, as we know that $S_{2}$ does not contribute to this equation.

In order to calculate the renormalized dissipation rate, we assume a power-law form for the spectrum $E(k)=\alpha \varepsilon^{r} k^{s}$. Then, the requirement that the renormalized viscosity (19) and its increment (20) scale in the same way, along with the conservation requirement of (23), yield $r=2 / 3$ and $s=-5 / 3$, along with an expression for the Kolmogorov prefactor $\alpha$ (see equation (92) in reference [6]).

The theoretical prediction of $\alpha$ can be taken as a measure of the predicted dissipation rate, and in Figure 1 we show the result of such calculations, with $\alpha$ iterating to a fixed point for several different starting conditions at one value of the spatial rescaling factor. The fixed point corresponds to the upper end of the inertial range and the value of $\alpha$ at the fixed point agrees well with the result obtained from numerical simulations. Figure 2 shows the fixed-point value of $\alpha$ for a range of spatial rescaling factors. It is of interest to note that the chain-dotted curve to the left depicts an earlier version of the theory, in which the conditional average was approximated by a band-filtered average «. The dashed line shows the result of working out the limiting form of the viscosity (the stochastic part) with scale separation [6], while invoking a Markovian approximation. The continuous line shows the effect of including the square brackets in (20) and gives rise to a prediction of $\alpha=1.62 \pm 0.02$ for $0.2<\eta<0.6$, where the bandwidth $\eta=1-h$. Incidentally, it is perhaps worth remarking that the limit $\eta \rightarrow 0$ (which one would expect in the microscopic case) does not exist for macroscopic turbulence. This is a consequence of an exact symmetry of the NSE: local energy transfer vanishes when the wavevector triad takes the form of an equilateral triangle.

Lastly, there is the question: how good is our perturbation calculation? The expansion, 
which we truncate, is in powers of $\lambda=R_{n}^{2}(k)$, with integrals over moments of the $\psi^{+}$(where $\left.\left|\psi^{+}\right|_{\mathrm{rms}} \leq 1\right)$. With the maximum value $\lambda=0.16$, this is a small parameter, but possibly not small enough for the truncation to qualify as a rational approximation [9]. Accordingly, we may have to rely on the properties of the moment expansion. Certainly, the next step is to work out the magnitude of the fourth-order term. This is the subject of current work.

Both authors acknowledge the support and facilities provided by the Isaac Newton Institute. We also thank Prof. M.E. Cates, G. Fullerton, A. Hunter and A. Quinn for reading the manuscript and making numerous helpful comments. CJ acknowledges the financial support of the Engineering and Physical Sciences Research Council. 


\section{FIGURES}

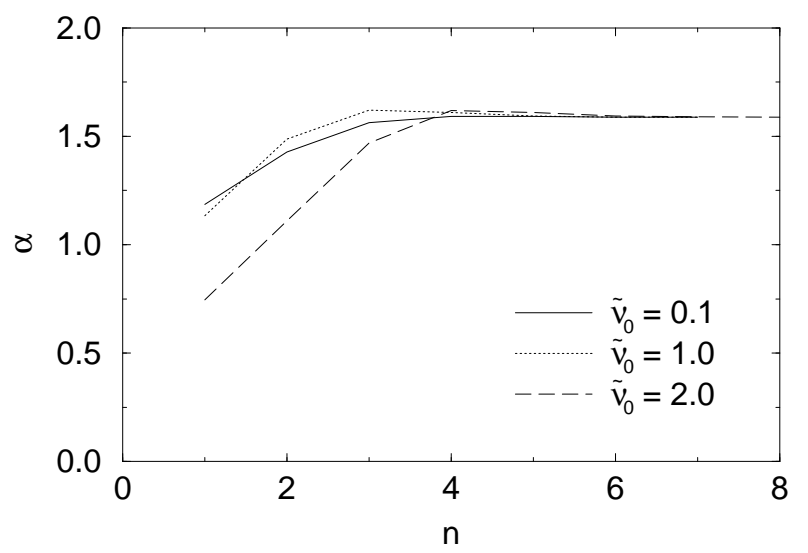

FIG. 1. The Kolmogorov prefactor $\alpha$ reaching a fixed point for a variety of starting viscosities $\tilde{\nu}_{0}$. (For the case where the spatial rescaling factor $h=0.60$ or the bandwidth $\eta=0.40$.)

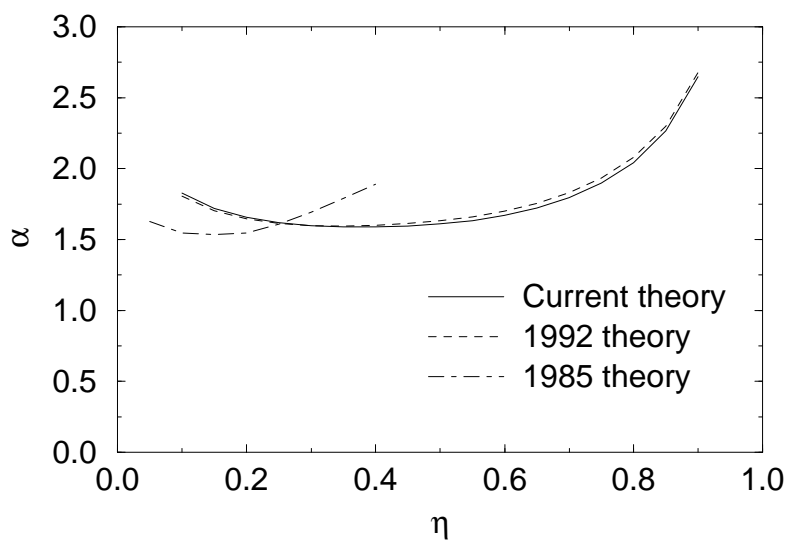

FIG. 2. Variation of the Kolmogorov prefactor $\alpha$ with bandwidth $\eta$ or spatial rescaling factor $h$. 


\section{REFERENCES}

[1] W. D. McComb and C. Johnston, J. Phys. A: Math. Gen 33, L15 (2000).

[2] E. A. Novikov, Fluid. Dyn. Res. 12, 107 (1993), J. A. Langford and R. D. Moser, J. Fluid Mech. 398, 321 (1999), T. Dubois, F. Jauberteau, and R. Temam, Dynamic multilevel methods and the numerical simulation of turbulence, Cambridge University Press, Cambridge, 1999.

[3] G. L. Eyink, Phys. Fluids 6, 3063 (1994).

[4] W. D. McComb, Application of Renormalization Group (RG) methods to the subgrid modelling problem, in Volume 15 of Notes on Numerical Fluid Mechanics, pp. 67-81, Braunschweig, 1985, Friedr. Vieweg \& Sohn.

[5] W. D. McComb, W. Roberts, and A. G. Watt, Phys. Rev. A 45, 3507 (1992).

[6] W. D. McComb and A. G. Watt, Phys. Rev. A 46, 4797 (1992).

[7] G. K. Batchelor, The Theory of Homogeneous Turbulence, Cambridge University Press, Cambridge, 2nd edition, 1971.

[8] A. Papoulis, Probability, Random Variables and Stochastic Processes, McGraw-Hill, New York, 3rd edition, 1991.

[9] M. Van Dyke, Perturbation Methods in Fluid Mechanics, Parabolic Press, Stanford, CA, 2nd edition, 1975. 